Supporting Information

\title{
Single Nanoparticle Activities in Ensemble: a Study on Pd Cluster Nanoportals for Electrochemical Oxygen Evolution Reaction
}

\author{
Abheek Datta ${ }^{\dagger *}$, Alexander J. Porkovich ${ }^{\dagger}$, Pawan Kumar ${ }^{\dagger}$, Giorgos Nikoulis ${ }^{\ddagger}$, Joseph \\ Kioseoglou ${ }^{\star}$ Toshio Sasaki ${ }^{\S}$, Stephan Steinhauer ${ }^{\perp}$, Panagiotis Grammatikopoulos ${ }^{\dagger *}$, \\ Mukhles Sowwan ${ }^{\dagger *}$ \\ $\dagger$ Nanoparticles by Design Unit, Okinawa Institute of Science and Technology (OIST) Graduate \\ University, 1919-1 Tancha, Onna-Son, Okinawa 904-0495, Japan \\ † Department of Physics, Aristotle University of Thessaloniki, GR - 54124 Thessaloniki, Greece \\ $\S$ Imaging Section, Okinawa Institute of Science and Technology (OIST) Graduate University, \\ 1919-1 Tancha, Onna-Son, Okinawa 904-0495, Japan \\ $\perp$ Department of Applied Physics, KTH Royal Institute of Technology, SE-106 91, Stockholm, \\ Sweden \\ Corresponding Authors \\ *mukhles@oist.jp \\ * pgrammatikopoulos@,oist.jp \\ * datta.abheek@oist.jp
}




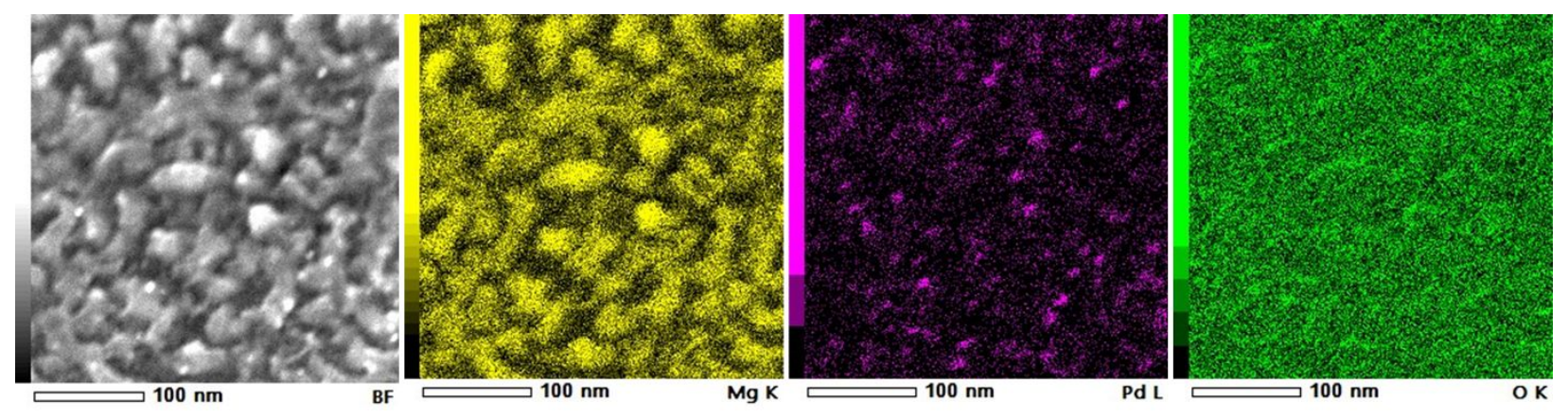

Figure S1: Exemplary elemental mapping of $\mathrm{Pd}^{3} \mathrm{Mg}^{20}$ obtained by EDX. 

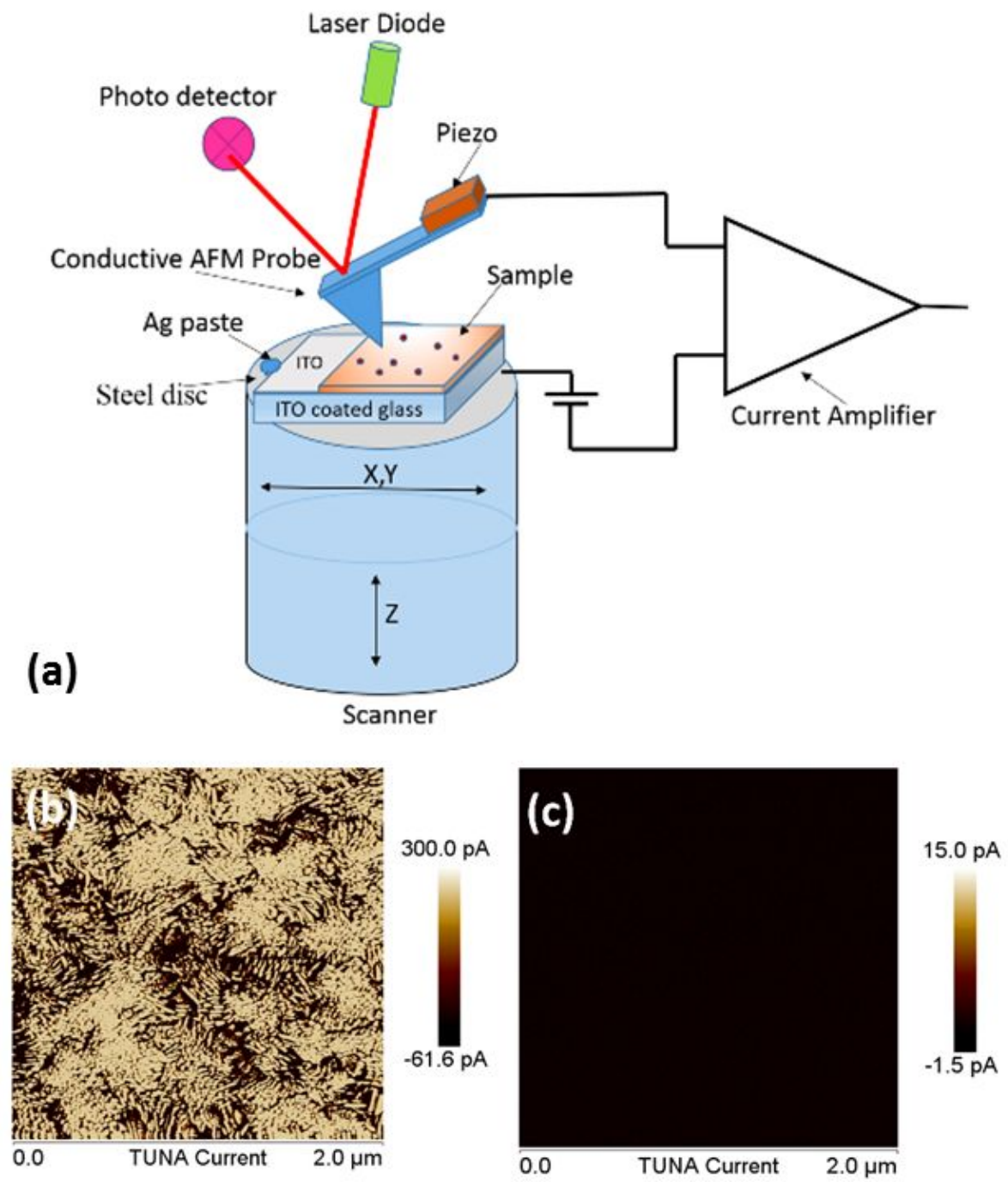

Figure S2: (a) Schematic representation of PF-TUNA working principle. PF-TUNA current images for (b) bare ITO and (c) MgO on ITO.
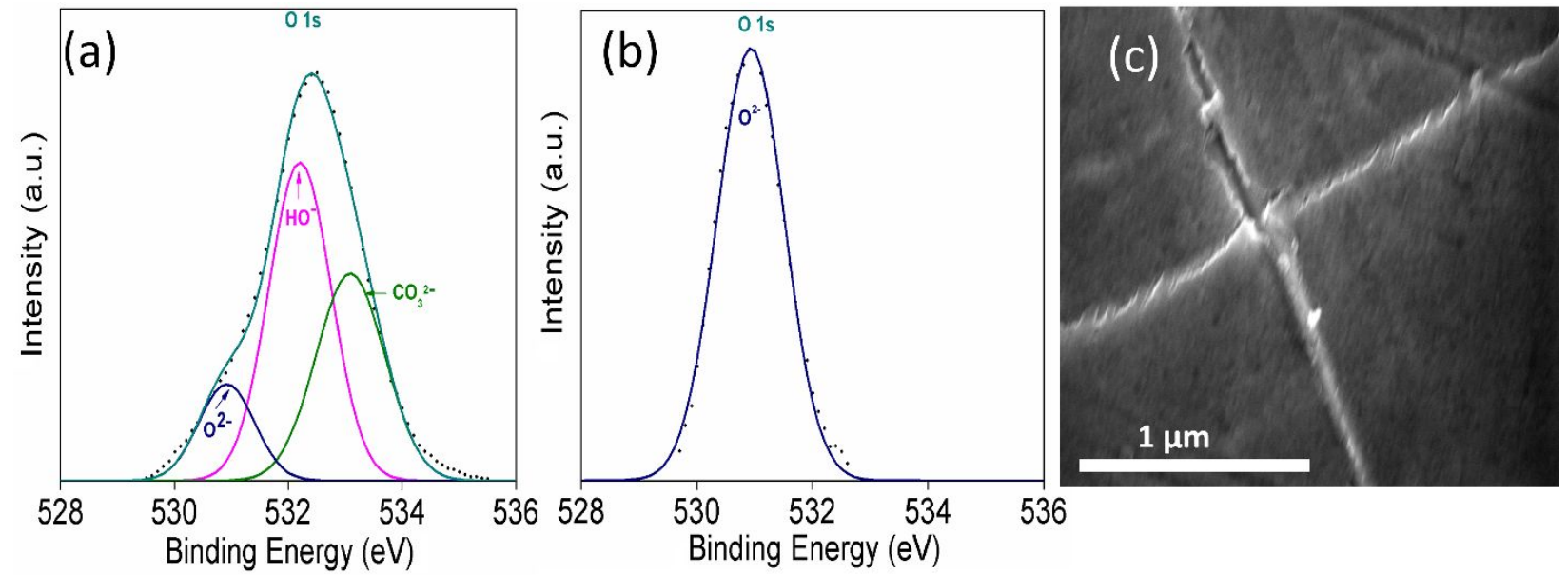

Figure S3: Core spectra of $O 1 \mathrm{~s}(\mathrm{a})$ at $15^{\text {th }}$ second and (b) at $350^{\text {th }}$ second of etching for $\mathrm{Pd}^{5} \mathrm{Mg}^{40}$. (c) SEM image of surface $\mathrm{Pd}^{5} \mathrm{Mg}^{40}$. 


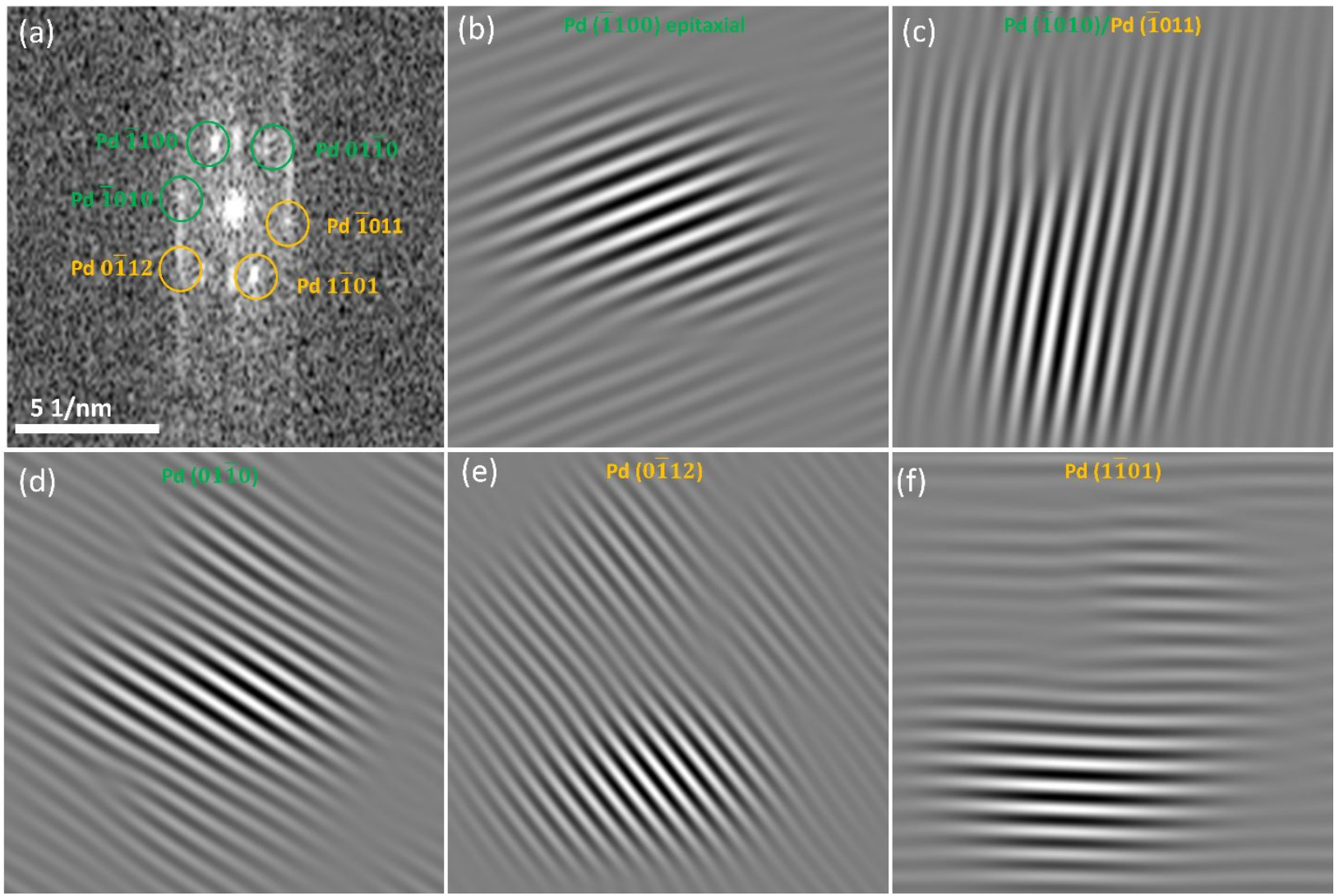

Figure S4: (a) FFT of the Pd particle shown in figure 4a of main article. (b), (c), (d), (e) and (f) display Bragg-filtered images of the spots from (a).

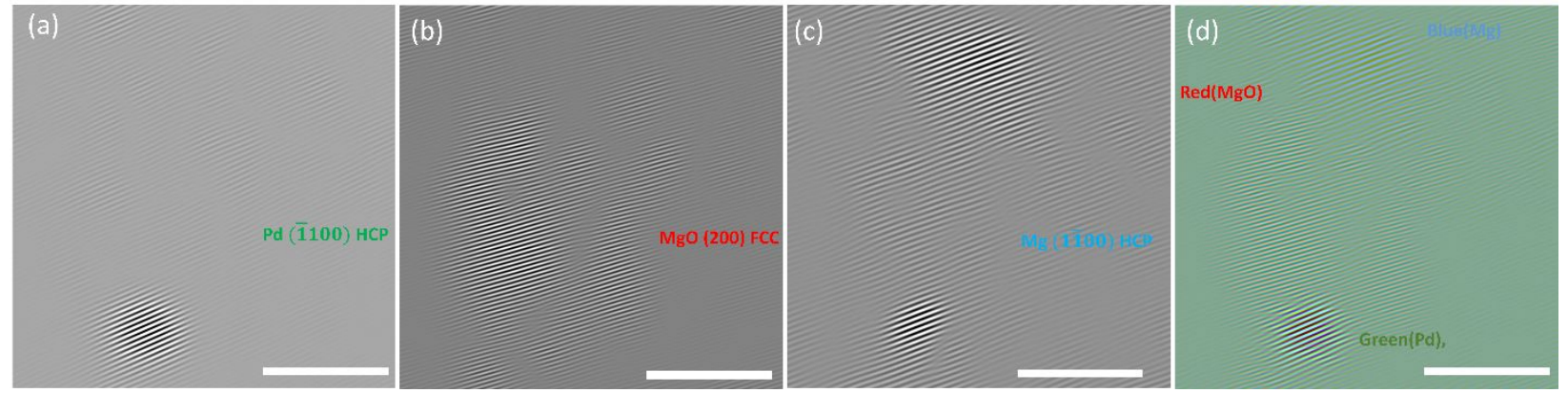

Figure S5: Bragg-filtered images for individual planes identified from figure 4a in main article for $\mathrm{Pd}(\mathrm{a}), \mathrm{MgO}$ (b) and $\mathrm{Mg}$ (c). (d) RGB color superimposition of the Bragg-filtered images. Scale bars indicate $5 \mathrm{~nm}$. 

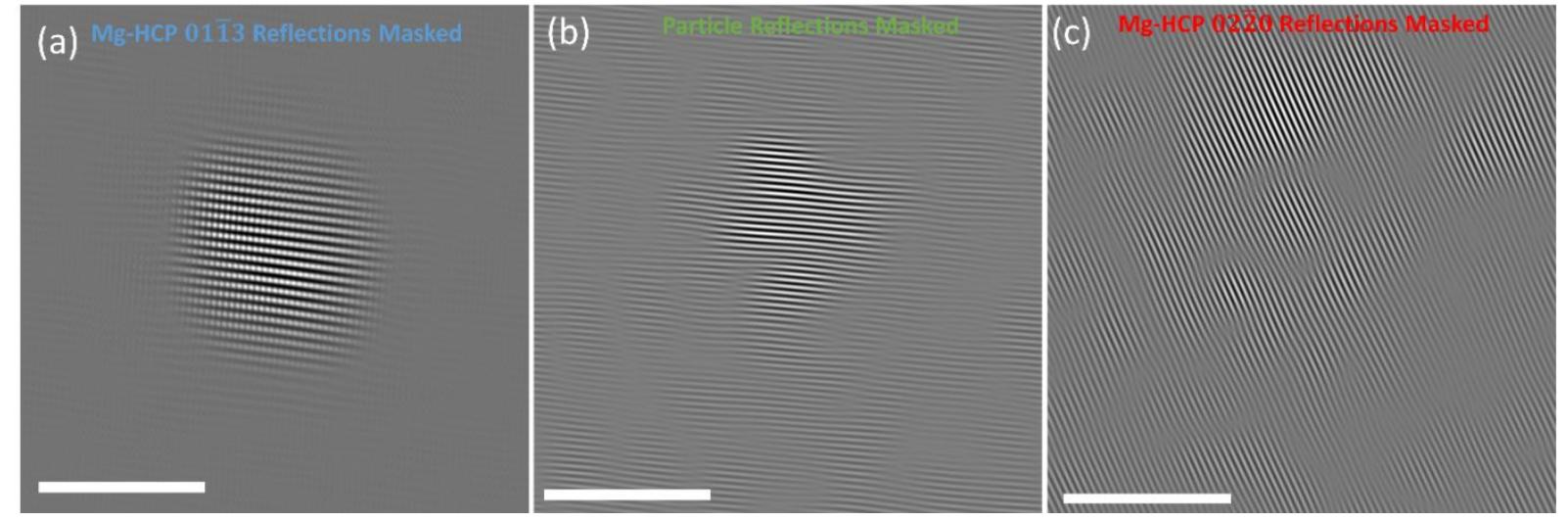

Figure S6: Bragg-filtered image of (a) the four spots indexed to Pd in the FFT for $\mathrm{Pd}^{5} \mathrm{Mg}^{20}$, (b) from the masking of the Mg-hcp 01133, and (c) derived from the Mg-hcp $02 \overline{2} 0$ reflections. Scale bars indicate $5 \mathrm{~nm}$.

(a)

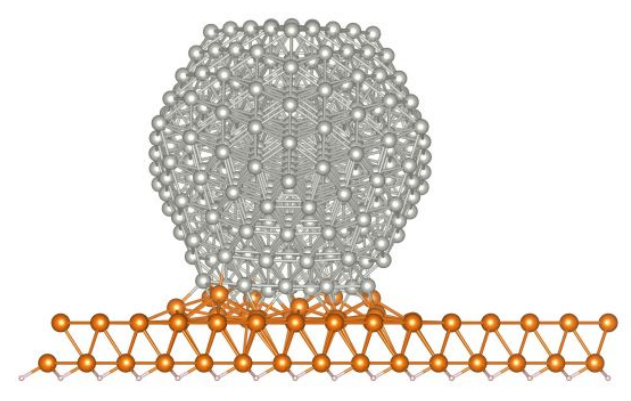

(b)

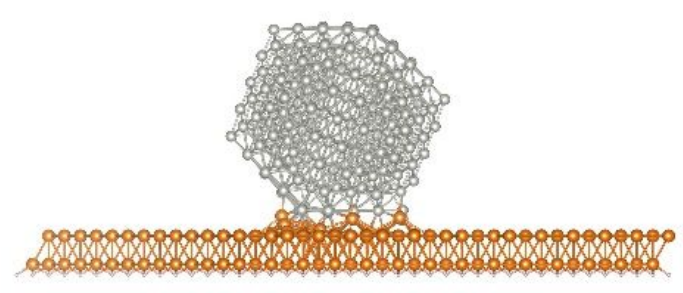


(c)

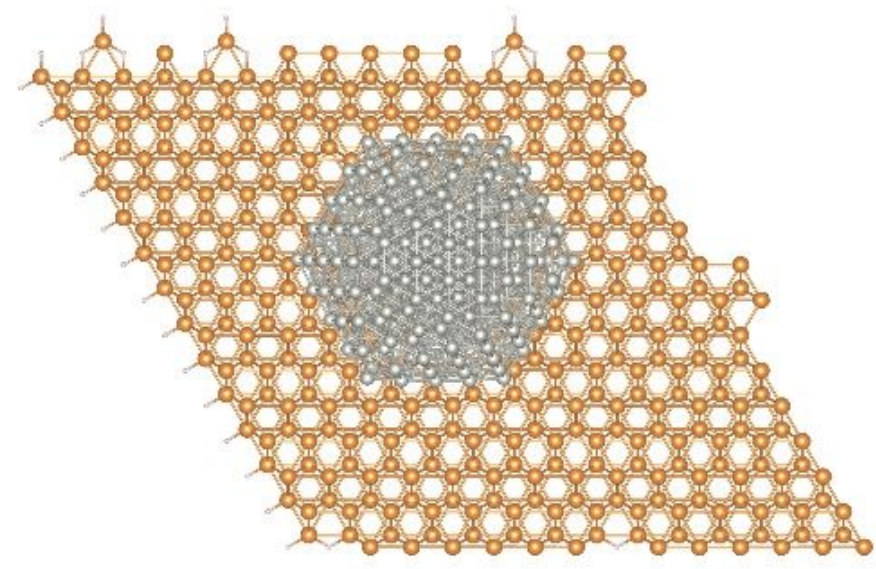

(d)

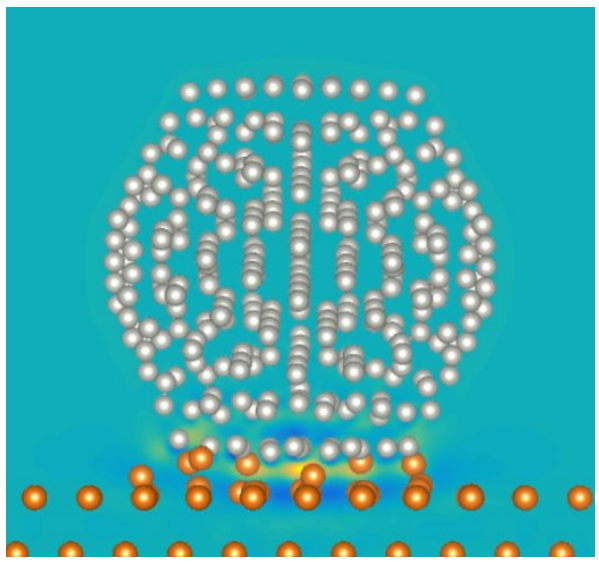

(e)

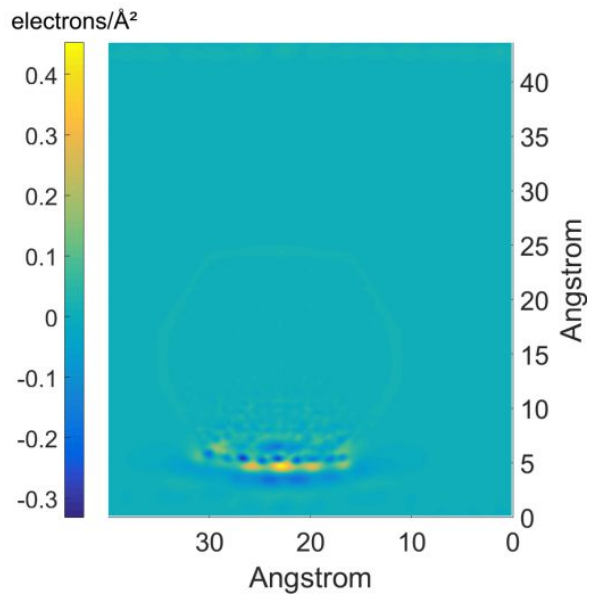

Figure S7: (a, b) Side views, (c) top view, and (d, e) charge density transfer plot of a relaxed system containing an icosahedral Pd NP with its (111) facet on top of the (0001) surface of the Mg support, with (d) and without (e) the relaxed atomistic model.

(a)

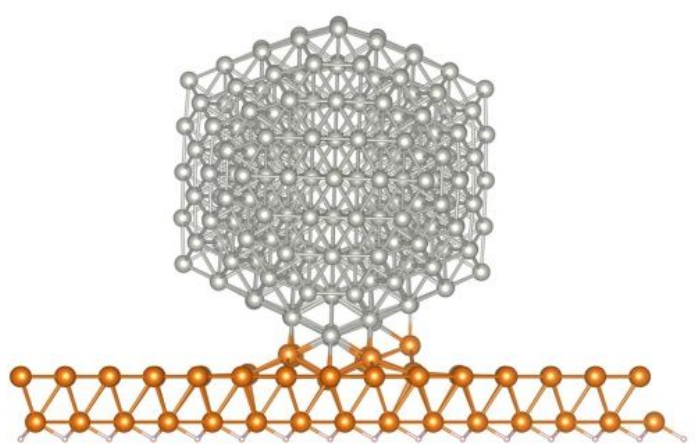

(b)

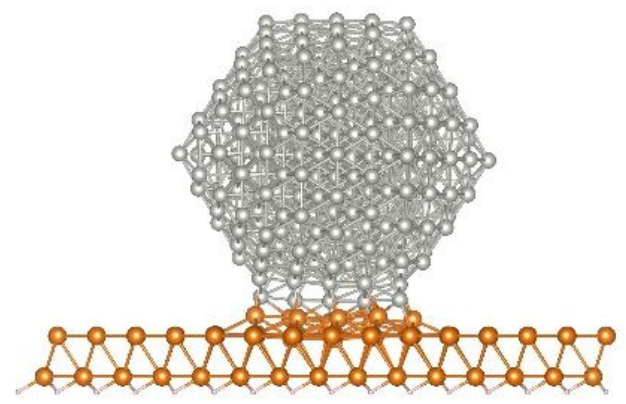


(c)

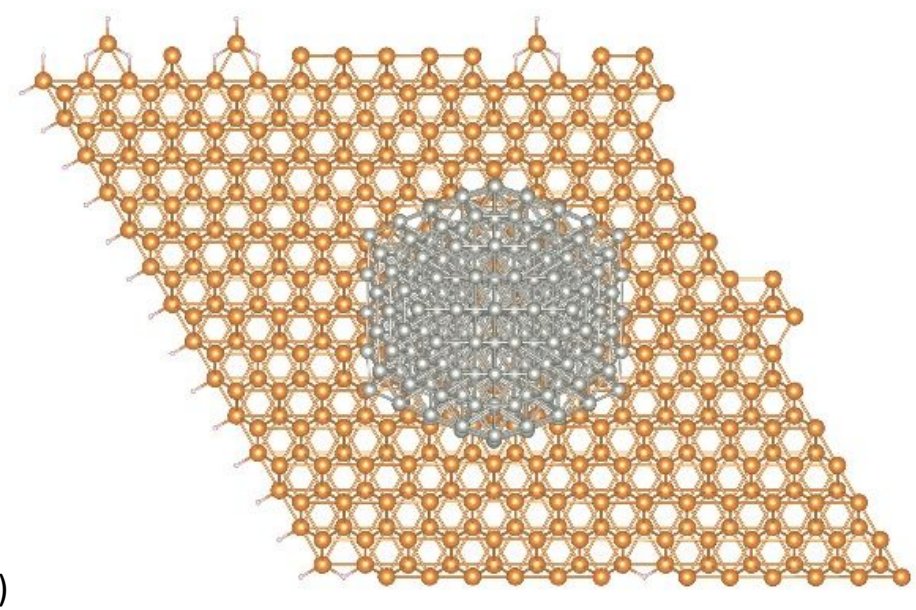

(d)

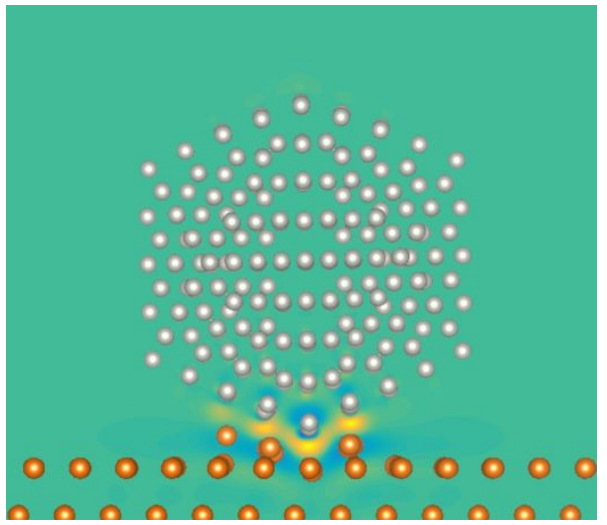

Figure S8: $(\mathrm{a}, \mathrm{b})$ Side views, (c) top view, and (d, e) charge density transfer plot of a relaxed system containing an icosahedral Pd NP with an edge at the intersection of $\{111\}$ planes on top of the (0001) surface of the Mg support, with (d) and without (e) the relaxed atomistic model.

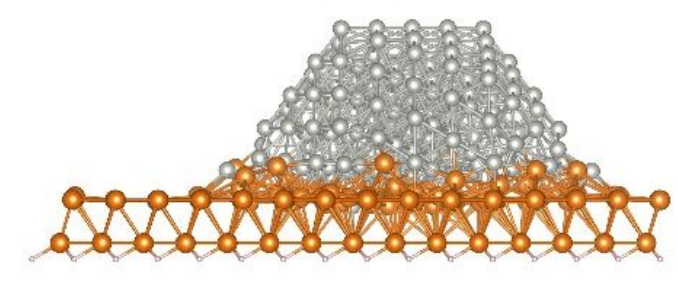

(a) (b)

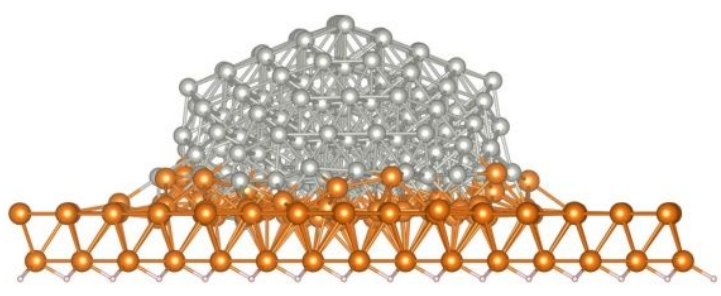




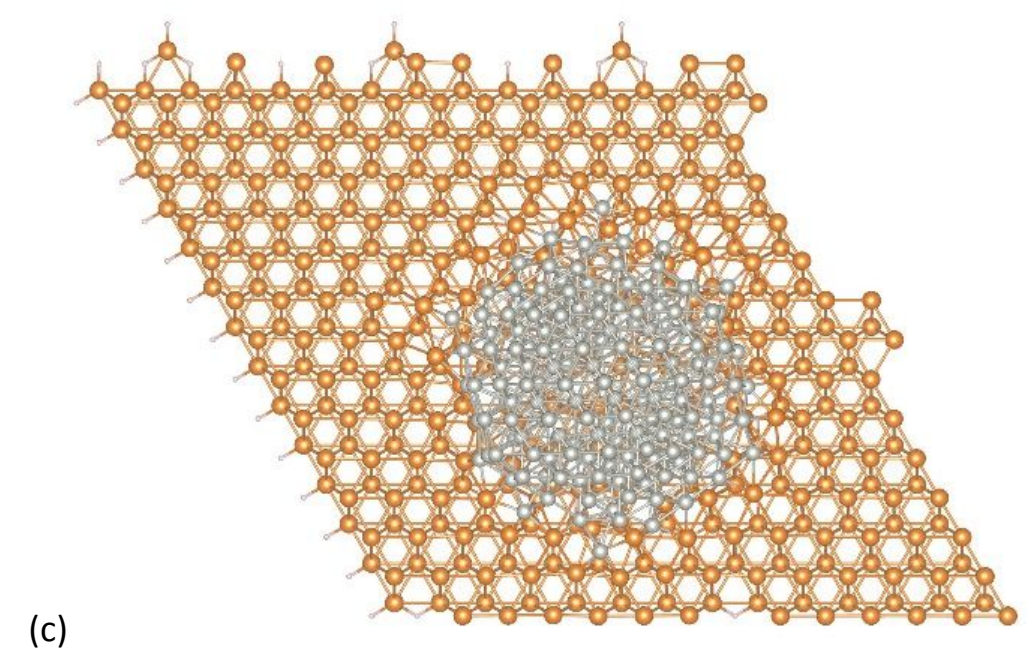

(c)

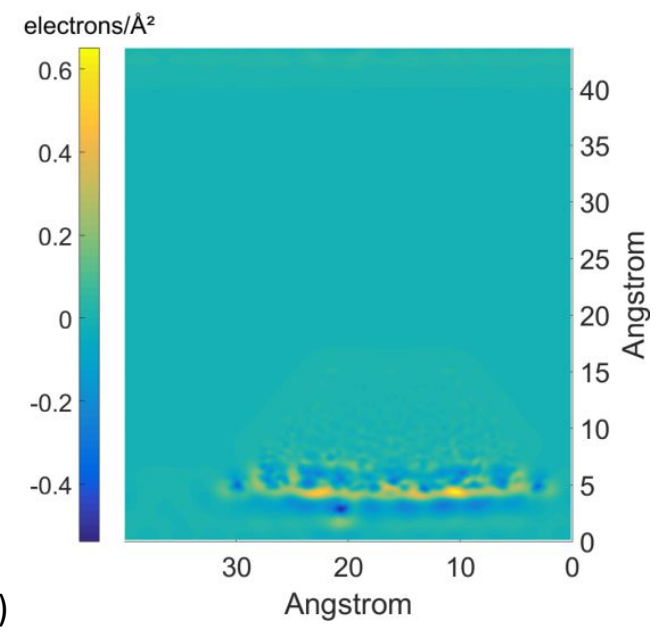

Figure S9: $(a, b)$ Side views, (c) top view, and (d) charge density transfer plot of a relaxed system containing an icosahedral Pd NP on top of the (0001) surface of the Mg support along the (a) projection, with similar orientation to that of figure S8, but with the NP truncated along its equatorial plane, instead.

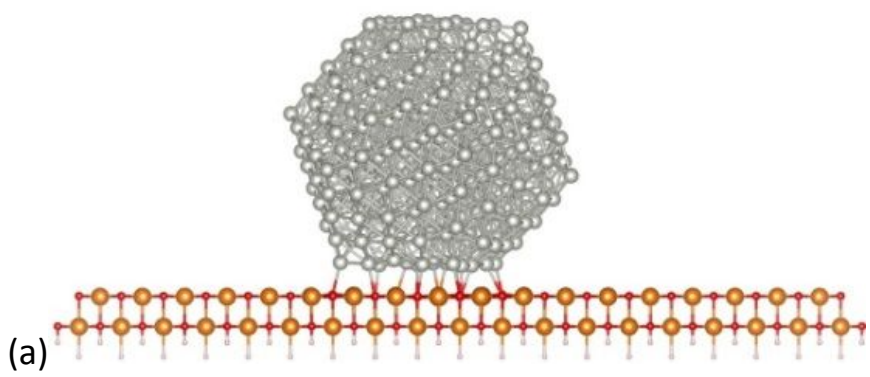

(b)

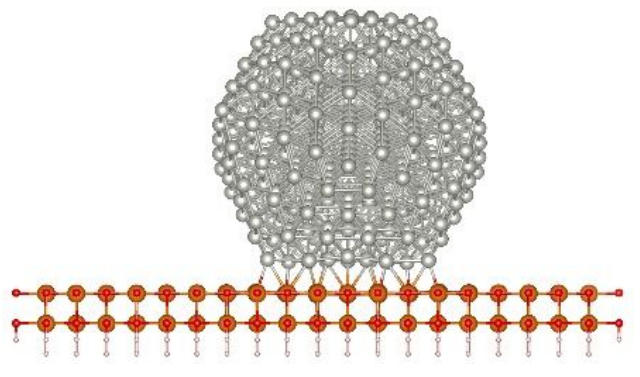


(c)

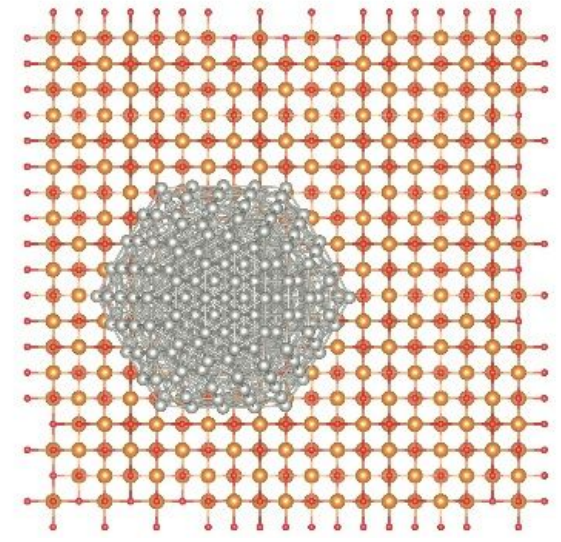

(e)
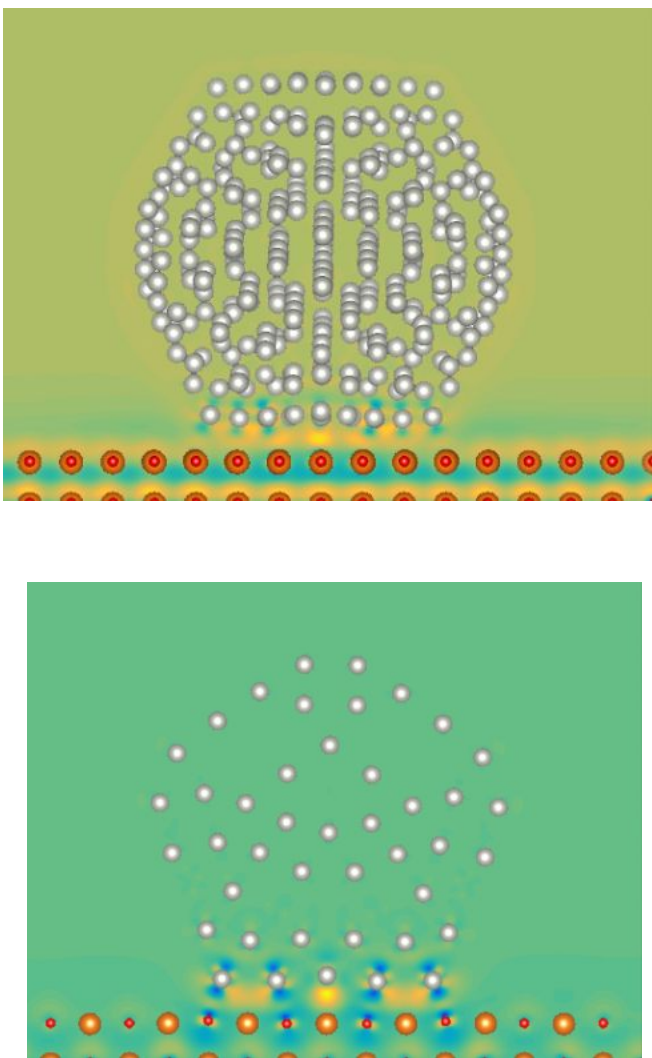

$(\mathrm{g})$ (d)

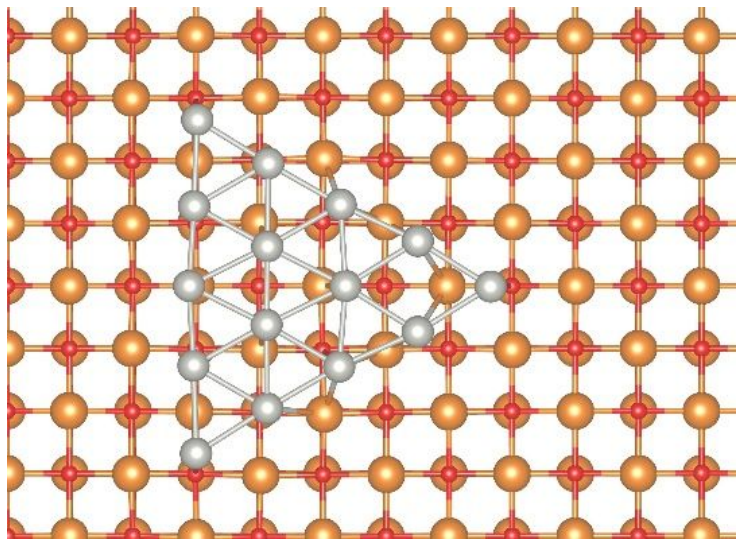

(f)
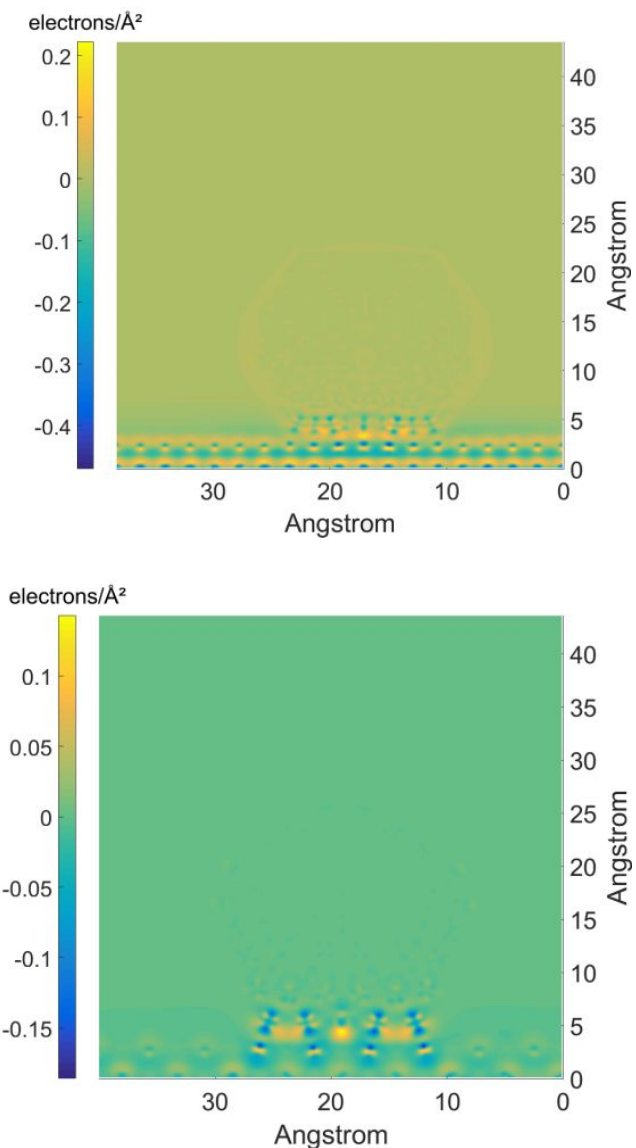

Figure S10: (a, b) Side views, (c) top view, (d) interface view, and charge density transfer plot with and without the atomistic model, (e) and (f), respectively, of the relaxed system along the (a) projection containing an icosahedral Pd NP with its (111) facet on top of the (100) surface of the $\mathrm{MgO}$ support. In (g) and (h) the charge density transfer plot is shown along the (b) projection (with and without the atomistic model, respectively) of a slice of the NP, in order to clarify the associated bond charges. 
(a)
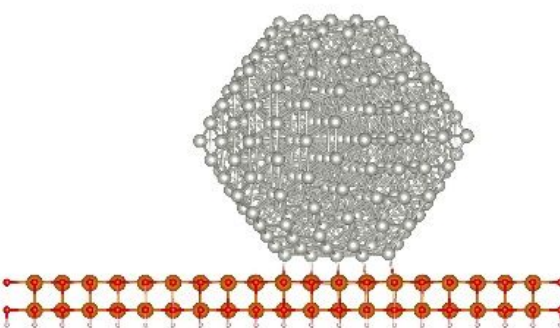

(c)
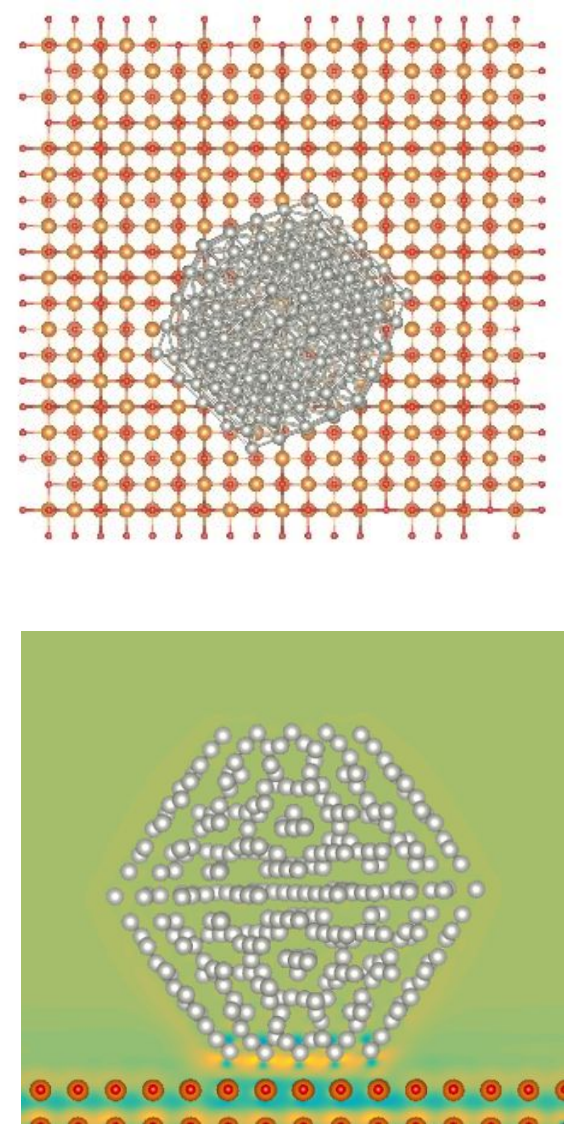

(e)

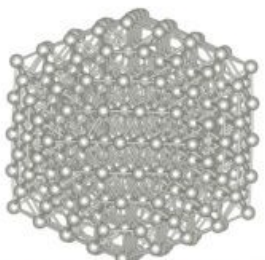

(b)

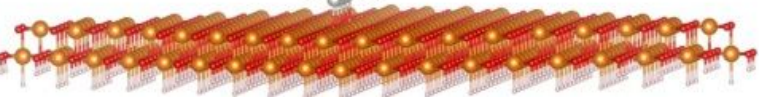

(d)
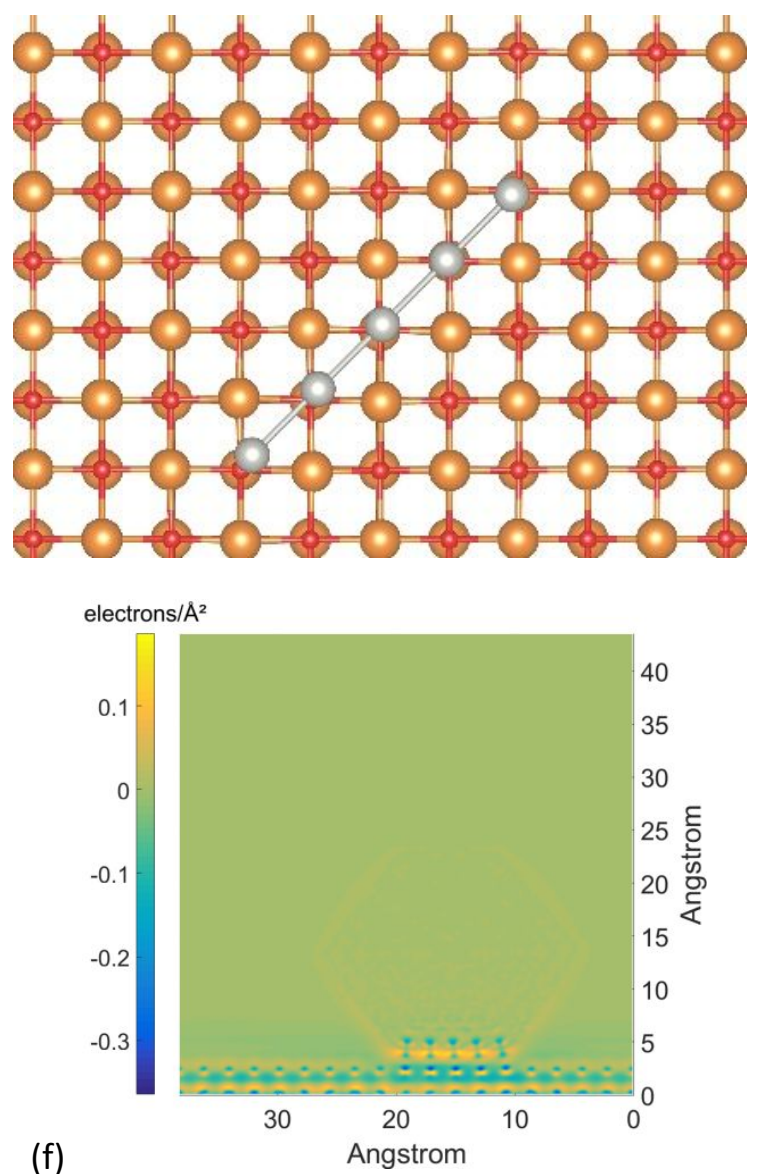

Figure S11: (a, b) Side views, (c) top view, (d) interface view, and charge density transfer plot with and without the atomistic model, (e) and (f), respectively, of a relaxed system along the (a) projection, containing an icosahedral Pd NP with an edge at the intersection of $\{111\}$ planes on top of the (100) surface of the $\mathrm{MgO}$ support. 
(a)

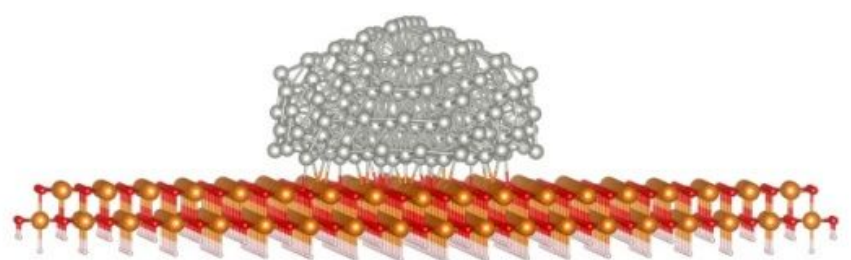

(c)
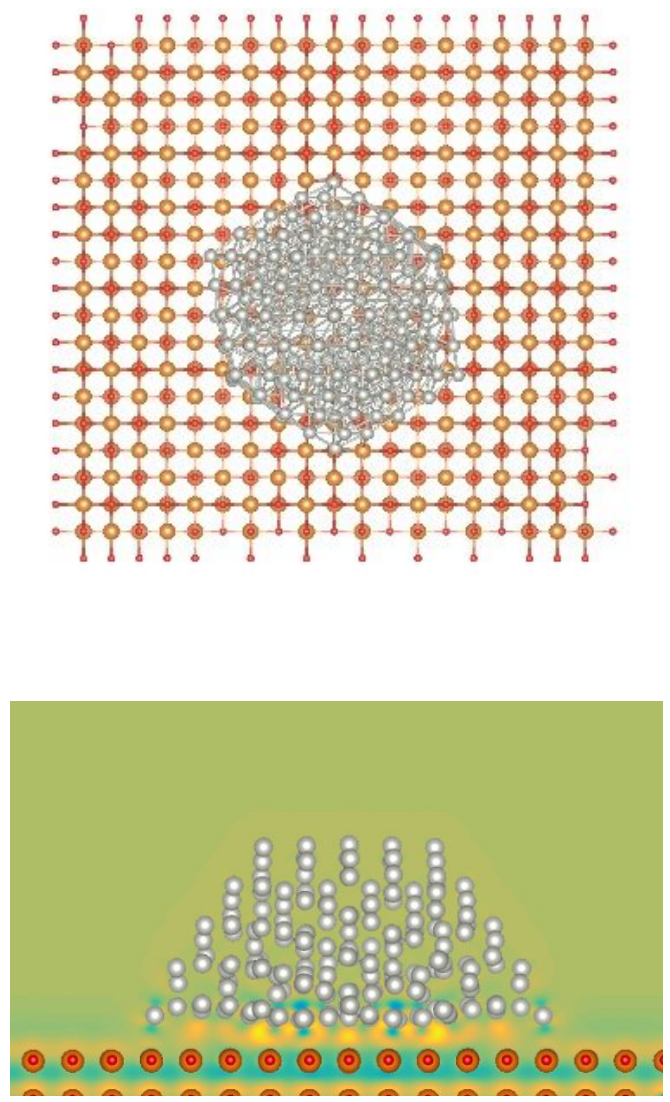

(e) (b)

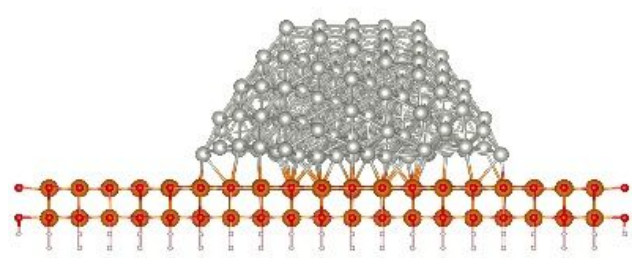

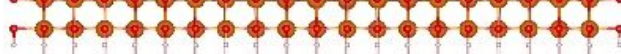
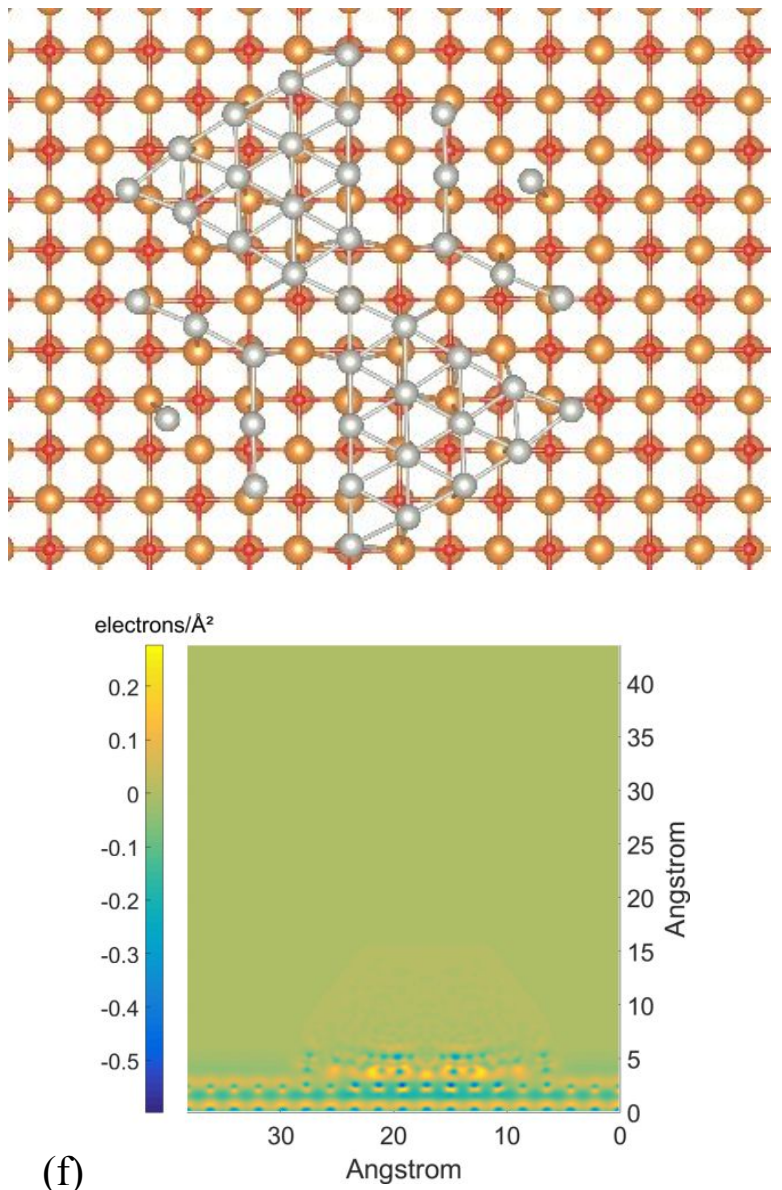

Figure S12: (a, b) Side views, (c) top view, (d) interface view, and charge density transfer plot with and without the atomistic model, (e) and (f), respectively, of a relaxed system containing an icosahedral Pd NP on top of the (100) surface of the MgO support with similar orientation to that of figure S11, but with the NP truncated along its equatorial plane, instead. 
(a)

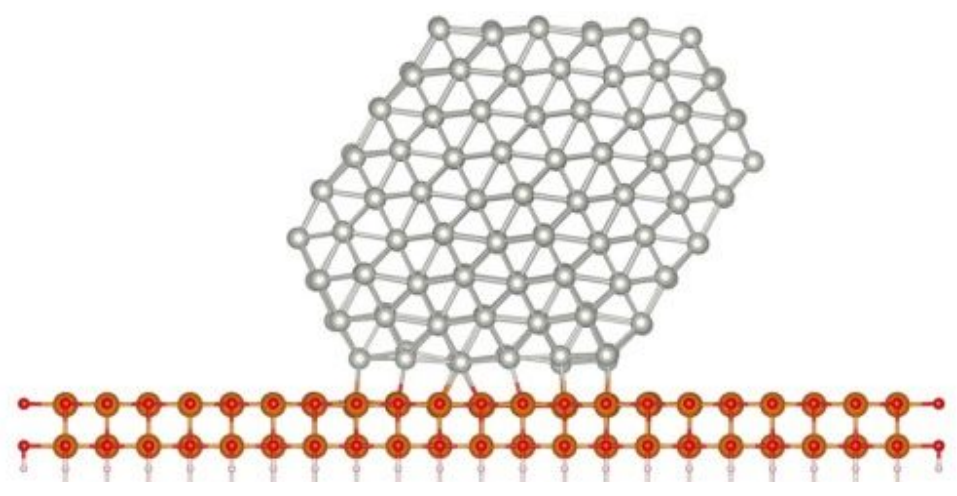

(b)
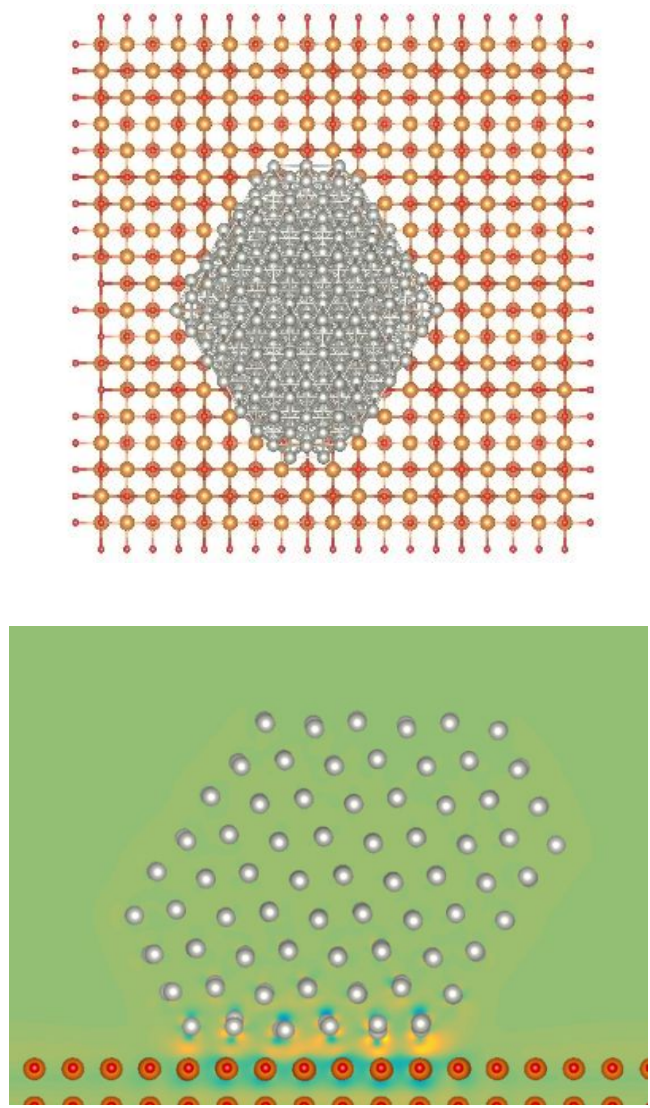

(d) (c)
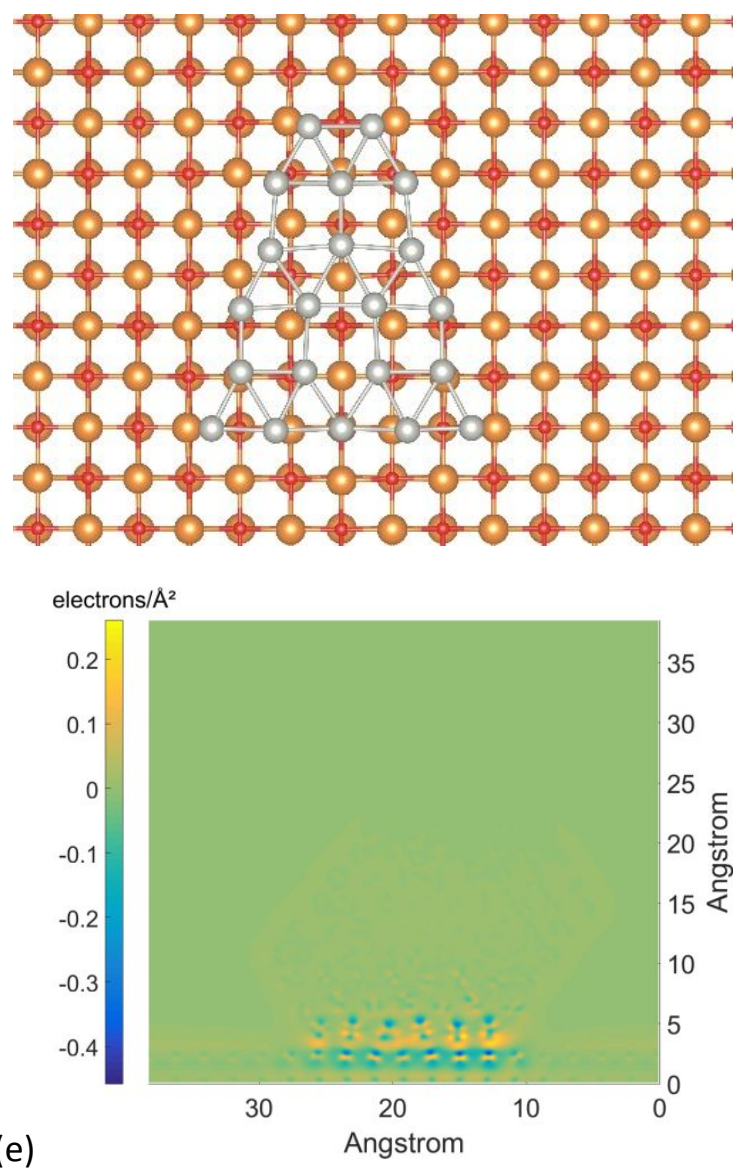

Figure S13: (a) Side view, (b) top view, (c) interface view, and charge density transfer plot with and without the atomistic model, (d) and (e), respectively, of a relaxed system containing a hexagonal Pd NP of $\{0001\}$ and $\{10 \overline{1} 1\}$ facets, with a (1011) facet on top of the (100) surface of the $\mathrm{MgO}$ support. 

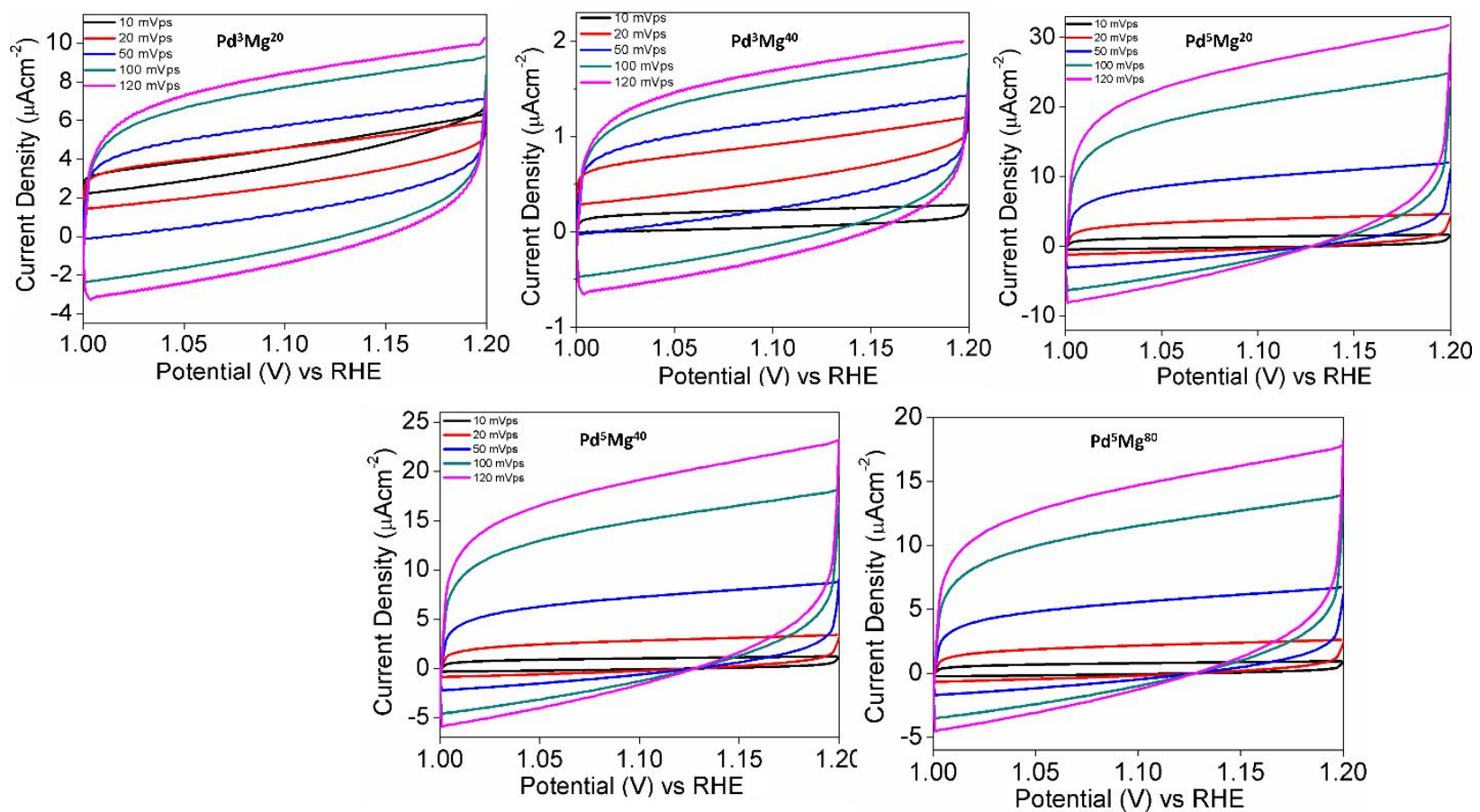

Figure S14: Cyclic voltammograms at non-faradic regions.
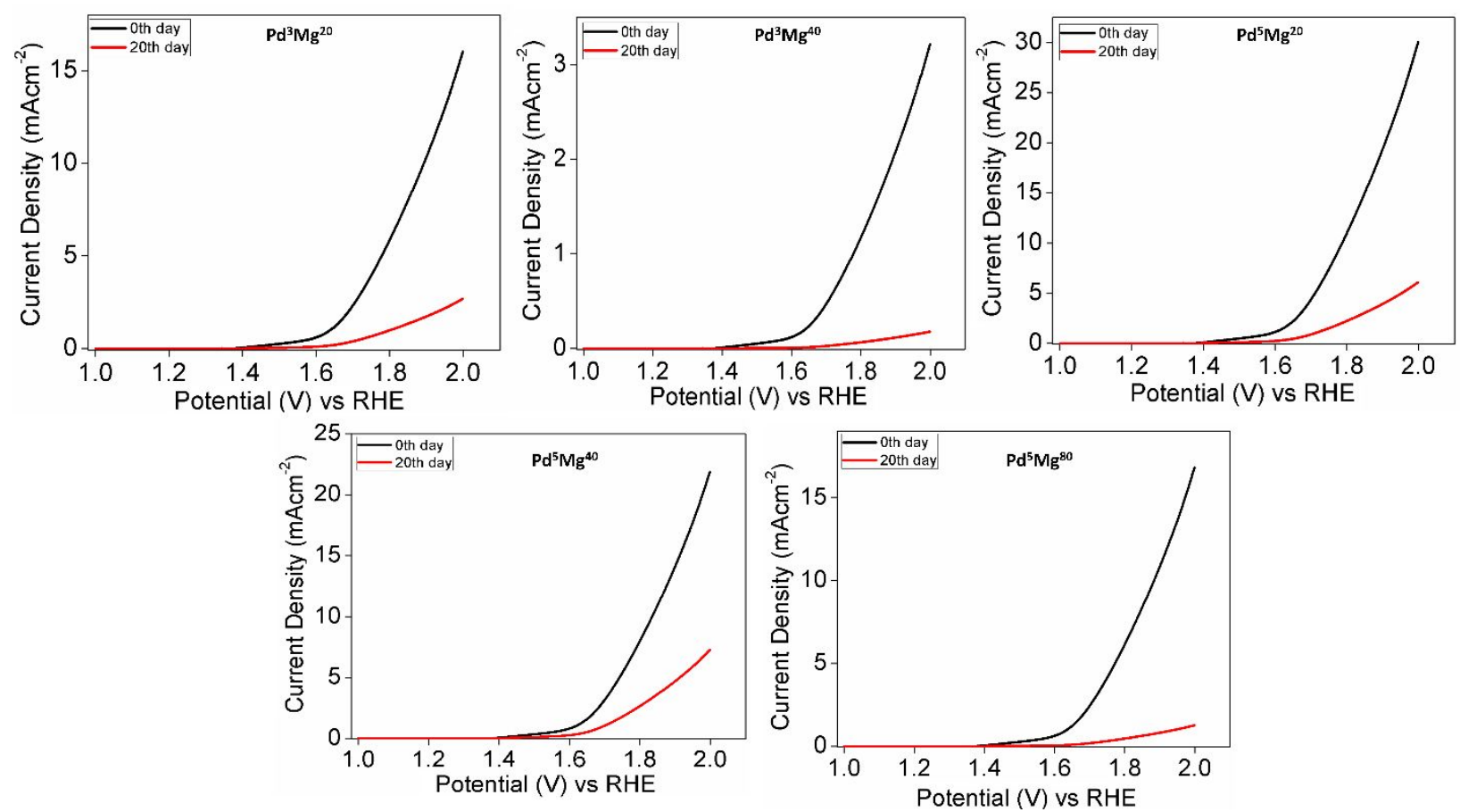

Figure S15: OER stability studies for catalysts 